\title{
Rotationally symmetric Ricci flow on asymptotically flat manifolds
}

\author{
Todd A. Oliynyk And ERIC WoOlgar
}

\begin{abstract}
We study Ricci flows on $\mathbb{R}^{n}, n \geq 3$, that evolve from rotationally symmetric, asymptotically flat initial data containing no embedded minimal hyperspheres. We show that in this case the flow is immortal, remains asymptotically flat, never develops a minimal hypersphere, and converges to flat Euclidean space as the time diverges to infinity. We discuss the behaviour of quasi-local mass under the flow, and relate this to a conjecture in string theory.
\end{abstract}

\section{Introduction}

The Ricci flow

$$
\frac{\partial g_{i j}}{\partial t}=-2 R_{i j}
$$

was first introduced in the mathematics literature by Richard Hamilton [10] in 1982. Almost immediately, it was applied to the classification problem for closed 3-manifolds and much subsequent work in the subject in the intervening 25 years has been focused on this application, culminating in the recent celebrated results of Perelman [20].

By contrast, Ricci flow on noncompact manifolds has received somewhat less attention. Of course, structures on noncompact manifolds, such as Ricci solitons, are relevant to the compact case, and this has been to now an important motivation for work on the noncompact case. The case of asymptotically flat Ricci flow has remained virtually untouched (nontrivial solitons do not occur in this case [17]).

But physics provides considerable motivation to study the asymptotically flat case. Our interest in it arises out of a conjectural scenario in string theory. Equation (1.1) is the leading-order renormalization group flow equation for a nonlinear sigma model that describes quantum strings propagating 
in a background spacetime [7]. ${ }^{1}$ What is important to understand from this statement is that fixed points of this equation provide geometric backgrounds in which the low energy excitations of quantum strings can propagate (in the approximation that radii of curvature are large and excitation energies small relative to the so-called string scale).

The variable $t$ in renormalization group flow is not time: it is (a constant times) the logarithm of the so-called renormalization scale. However, there are conjectured relationships between renormalization group flow and temporal evolution. A specific case concerns tachyon condensation, the scenario wherein an unstable string system is balanced at the top of a hill of potential energy (for a review of tachyon condensation, see [13]). The system falls off the hill, radiating away energy in gravitational waves. The system comes to rest in a valley representing a stable minimum of potential energy. In open string theory, a more elaborate version of this scenario involving the evaporation of a brane and the formation of closed strings is now well understood, even quantitatively. In closed string theory, much less is known but, conjecturally, the fixed points of the renormalization group flow equation (1.1) are the possible endpoints of this evolution. Sometimes it is further conjectured that time evolution in closed string theory near the fixed points is determined by renormalization group flow, and then $t$ in (1.1) does acquire an interpretation as a time.

Comparing dynamical and renormalization group pictures, we see that the radiation of positive energy in the form of gravitational waves as the system comes to rest in the valley should produce a corresponding decrease in the mass of the manifold under the Ricci flow. This suggests that we should endeavour to formulate and test a conjecture that mass decreases under Ricci flow, at least if the initial mass is positive.

The asymptotically flat case has a well-defined notion of mass, the Arnowitt-Deser-Misner (ADM) mass, so this seems an appropriate setting in which to formulate the conjecture. However, the metric entering the renormalization group flow or Ricci flow in this scenario is not the full spacetime metric, for which (1.1) would not be even quasi-parabolic, but rather the induced Riemannian metric on a suitable spacelike submanifold [9]. Now ADM mass is conserved (between Cauchy surfaces, and in the closed string scenario of [9]), even in the presence of localized sources of radiation. This, we will see, is reflected in the Ricci flow. The mass of $g$ will not change during evolution by (1.1). But if energy loss through gravitational radiation

\footnotetext{
${ }^{1}$ We ignore the dilaton since it can be decoupled from the metric in renormalization group flow [18].
} 
occurs, then the quasi-local mass contained within a compact region should change along the flow to reflect this. ${ }^{2}$

After describing asymptotically flat manifolds in Section 2, we outline local existence, uniqueness, and continuation for Ricci flow on asymptotically flat manifolds in Section 3. We then specialize to rotational symmetry in Section 4. In Section 4.1, we pass to a coordinate system well suited to our subsequent assumption that no minimal hyperspheres are present initially. We show in Section 4.3 that this coordinate system remains well defined on the interval $\left[0, T_{M}\right)$, where $T_{M}$ is the maximal time of existence for Ricci flow. This is essentially a consequence of the result, proved in Section 4.2, that no minimal hyperspheres develop during the flow.

The absence of minimal spheres allows us to analyze the problem in terms of a single PDE, the master equation (4.18). From this equation, we derive a number of maximum principles that yield uniform bounds on the curvature which allow us to conclude that $T_{M}=\infty$. We obtain these principles in the first two subsections of Section 5. Even better, we obtain not just uniform bounds but decay estimates, from which we can prove convergence to flat Euclidean space. Now given our assumptions, this is the only Ricci-flat fixed point available. That is, the string theory discussion above would lead one to conjecture that:

When no minimal hypersphere is present, rotationally symmetric, asymptotically flat Ricci flow is immortal and converges to flat space as $t \rightarrow \infty$;

and this is what we show. Though we have motivated this conjecture from string theory for the case of positive initial mass, we will prove that it holds whether or not the initial mass is positive. This is our main theorem, proved in Section 5.3, which states:

Theorem 1.1. Let $\left\{x^{i}\right\}_{i=1}^{n}$ be a fixed Cartesian coordinate system on $\mathbb{R}^{n}$, $n \geq 3$. Let $\hat{g}=\hat{g}_{i j} d x^{i} d x^{j}$ be an asymptotically flat, rotationally symmetric metric on $\mathbb{R}^{n}$ of class $H_{\delta}^{k}$ with $k>n / 2+4$ and $\delta<0$. If $\left(\mathbb{R}^{n}, \hat{g}\right)$ does not contain any minimal hyperspheres, then there exists a solution $g(t, x) \in$ $C^{\infty}\left((0, \infty) \times \mathbb{R}^{n}\right)$ to Ricci flow (1.1) such that

(i) $g(0, x)=\hat{g}(x)$,

\footnotetext{
${ }^{2}$ We prefer not to discuss in terms of the Bondi mass, which would require us to pass back to the Lorentzian setting which is not our focus in this article. See [9] for a discussion in terms of Bondi mass.
} 
(ii) $g_{i j}-\delta_{i j} \in C^{1}\left([0, T], H_{\delta}^{k-2}\right) \quad$ and $g_{i j}-\delta_{i j} \in C^{1}\left(\left[T_{1}, T_{2}\right], H_{\delta}^{\ell}\right)$ for any $0<T_{1}<T_{2}<\infty, 0<T<\infty, \ell \geq 0$,

(iii) for each integer $\ell \geq 0$ there exists a constant $C_{\ell}>0$ such that

$$
\sup _{x \in \mathbb{R}^{n}}\left|\nabla^{\ell} \operatorname{Rm}(t, x)\right|_{g(t, x)} \leq \frac{C_{\ell}}{(1+t) t^{\ell / 2}} \forall t>0
$$

(iv) the flow converges to $n$-dimensional Euclidean space $\mathbb{E}^{n}$ in the pointed Cheeger-Gromov sense as $t \rightarrow \infty$, and

(v) if furthermore $k>n / 2+6, \delta<\min \{4-n, 1-n / 2\}, \hat{R} \geq 0$, and $\hat{R} \in$ $L^{1}$, then the ADM mass of $g(t)$ is well defined and mass $(g(t))=$ mass $(\hat{g})$ for all $t \geq 0$.

When a minimal hypersphere is present initially, if the neck is sufficiently pinched then we expect long-time existence to fail. To see why, consider rotationally symmetric metrics on $S^{n}$. If there is a sufficiently pinched minimal $(n-1)$-sphere, the curvature blows up in finite time. This has been shown both rigorously $(n \geq 3)$ [1] and numerically $(n=3)$ [8]. Our assumption of no minimal spheres in the initial data is intended to prevent this. The ability to make this assumption and to choose coordinates adapted to it is a distinct advantage of the noncompact case. However, we also expect (based, e.g., on [8]) that for initial data with minimal hyperspheres that have only a mild neck pinching, the flow will continue to exist globally in time as well. Thus, when a minimal hypersphere is present, we believe there would be considerable interest in determining a precise criterion for global existence in terms of the degree of neck pinching because of the possibility, raised in [8], that the critical case on the border between singularity formation and immortality may exhibit universal features such as those observed in critical collapse in general relativity [5].

The constancy of the ADM mass in statement (v) is not at odds with the conclusion that the flow converges to a flat and therefore massless manifold. This constancy was also noted in [6] but we draw different conclusions concerning the limit manifold, owing to our use of the pointed CheegerGromov sense of convergence of Riemannian manifolds. ${ }^{3}$ In Section 4.4

\footnotetext{
${ }^{3}$ The rotationally symmetric, expanding soliton of [9] can be used to illustrate this phenomenon explicitly (albeit in 2 dimensions, whereas our results are for $n \geq 3$ dimensions). For this soliton, one can easily compute the Brown-York quasilocal mass on any ball whose proper radius is fixed in time and see that for each such ball the quasi-local mass tends to zero as $t \rightarrow \infty$, and the flow converges to
} 
we define three different kinds of metric balls in $\left(\mathbb{R}^{n}, g(t)\right), n \geq 3$; balls of fixed radius, fixed volume, and fixed surface area of the bounding hypersphere. To clarify the behaviour of the mass in the limit $t \rightarrow \infty$, we express the Brown-York quasi-local mass of these balls in terms of sectional curvature and, by anticipating the decay rate for sectional curvature derived in Section 5, show that these quasi-local masses go to zero as $t \rightarrow \infty$, even though the ADM mass, as measured at infinity, is constant. The picture is not strongly dependent on the definition of quasi-local mass, of which the Brown-York definition is but one among many. In rotational symmetry in any dimension, the metric has only one "degree of freedom". The study of the evolution of quasi-local mass then reduces to the study of this single degree of freedom, no matter which definition of quasi-local mass one prefers. ${ }^{4}$

The problem of global existence for rotationally symmetric metrics on $\mathbb{R}^{3}$ has previously been investigated in [14]. There the assumptions on the initial metric are different than ours. Namely, the initial metric in [14] has positive sectional curvature and the manifold opens up as least as fast as a paraboloid. Under these assumptions, it is shown that Ricci flow exists for all future times and converges to either a flat metric or a rotationally symmetric Ricci soliton.

Finally, throughout we fix the dimension of the manifold to be $n \geq 3$. As well, we usually work with the Hamilton-DeTurck form of the Ricci flow

$$
\frac{\partial g_{i j}}{\partial t}=-2 R_{i j}+\nabla_{i} \xi_{j}+\nabla_{j} \xi_{i}
$$

which is obtained from the form (1.1) by allowing the coordinate basis in which $g_{i j}$ is written to evolve by a $t$-dependent diffeomorphism generated by the vector field $\xi$.

Euclidean 2-space. But the mass at infinity of the soliton (the deficit angle of the asymptotic cone in 2 dimensions) is a constant of the motion which can be set by initial conditions to take any value.

${ }^{4}$ The assumption of spherical symmetry in general relativity precludes gravitational radiation, according to the Birkhoff theorem. But on the string side of our scenario, the picture is one of closed strings existing as perturbations that break the spherical symmetry of the background metric (as well, we should include a dilaton background field that modifies general relativity). Viewed in the string picture, these perturbations create the radiation that is detected as a change in the quasi-local mass of the spherically symmetric Ricci flow. 


\section{Asymptotically flat manifolds}

The definition of asymptotically flat manifolds that we employ requires the use of weighted Sobolev spaces, which we will now define. Let $V$ be a finitedimensional vector space with inner product $(\cdot \mid \cdot)$ and corresponding norm $|\cdot|$. For $u \in L_{\mathrm{loc}}^{p}\left(\mathbb{R}^{n}, V\right), 1 \leq p \leq \infty$, and $\delta \in \mathbb{R}$, the weighted $L^{p}$ norm of $u$ is defined by

$$
\|u\|_{L_{\delta}^{p}}:= \begin{cases}\left\|\sigma^{-\delta-n / p} u\right\|_{L^{p}} & \text { if } 1 \leq p<\infty \\ \left\|\sigma^{-\delta} u\right\|_{L^{\infty}} & \text { if } p=\infty\end{cases}
$$

with

$$
\sigma(x):=\sqrt{1+|x|^{2}} .
$$

The weighted Sobolev norms are then given by

$$
\|u\|_{W_{\delta}^{k, p}}:= \begin{cases}\left(\sum_{|I| \leq k}\left\|D^{I} u\right\|_{L_{\delta-|I|}^{p}}^{p}\right)^{1 / p} & \text { if } 1 \leq p<\infty \\ \sum_{|I| \leq k}\left\|D^{I} u\right\|_{L_{\delta-|I|}^{\infty}} & \text { if } p=\infty\end{cases}
$$

where $k \in \mathbb{N}_{0}, I=\left(I_{1}, \ldots, I_{n}\right) \in \mathbb{N}_{0}^{n}$ is a multi-index and $D^{I}=\partial_{1}^{I_{1}} \ldots \partial_{n}^{I_{n}}$. Here $\partial_{i}=\left(\partial / \partial x^{i}\right)$ and $\left(x^{1}, \ldots, x^{n}\right)$ are the standard Cartesian coordinates on $\mathbb{R}^{n}$. The weighted Sobolev spaces are then defined as

$$
W_{\delta}^{k, p}=\left\{u \in W_{\mathrm{loc}}^{k, p}\left(\mathbb{R}^{n}, V\right) \mid\|u\|_{W_{\delta}^{k, p}}<\infty\right\} .
$$

In the case $p=2$, we will use the alternative notation $H_{\delta}^{k}=W_{\delta}^{k, 2}$.

As with the Sobolev spaces, we can define weighted version of the bounded $C^{k}$ function spaces $C_{b}^{k}:=C^{k}\left(\mathbb{R}^{n}, V\right) \cap W^{k, \infty}$ spaces. For a map $u \in C^{0}\left(\mathbb{R}^{n}, V\right)$ and $\delta \in \mathbb{R}$, let

$$
\|u\|_{C_{\delta}^{0}}:=\sup _{x \in \mathbb{R}^{n}}\left|\sigma(x)^{-\delta} u(x)\right|
$$

Using this norm, we define the $\|\cdot\|_{C_{\delta}^{k}}$ norm in the usual way:

$$
\|u\|_{C_{\delta}^{k}}:=\sum_{|I| \leq k}\left\|\partial^{I} u\right\|_{C_{\delta-|I|}^{0}} .
$$


Rotationally symmetric Ricci flow on asymptotically flat manifolds 541

So then

$$
C_{\delta}^{k}:=\left\{u \in C^{k}\left(\mathbb{R}^{n}, V\right) \mid\|u\|_{C_{\delta}^{k}}<\infty\right\} .
$$

We are now ready to define asymptotically flat manifolds.

Definition 2.1. Let $M$ be a smooth, connected, $n$-dimensional manifold, $n \geq 3$, with a Riemannian metric $g$ and let $E_{R}$ be the exterior region $\{x \in$ $\left.\mathbb{R}^{n}|| x \mid>R\right\}$. Then for $k>n / 2$ and $\delta<0,(M, g)$ is asymptotically flat of class $H_{\delta}^{k}$ if

(i) $g \in H_{\mathrm{loc}}^{k}(M)$,

(ii) there exists a finite collection $\left\{U_{\alpha}\right\}_{\alpha=1}^{m}$ of open subsets of $M$ and diffeomorphisms $\Phi_{\alpha}: E_{R} \rightarrow U_{\alpha}$ such that $M \backslash \cup_{\alpha} U_{\alpha}$ is compact, and

(iii) for each $\alpha \in\{1, \ldots, m\}$, there exists an $R>0$ such that $\left(\Phi_{\alpha}^{*} g\right)_{i j}-$ $\delta_{i j} \in H_{\delta}^{k}\left(E_{R}\right)$, where $\left(x^{1}, \ldots, x^{n}\right)$ are standard Cartesian coordinates on $\mathbb{R}^{n}$ and $\Phi_{\alpha}^{*} g=\left(\Phi_{\alpha}^{*} g\right)_{i j} d x^{i} d x^{j}$.

The integer $m$ counts the number of asymptotically flat "ends" of the manifold $M$. As discussed in the introduction, we are interested in manifolds where $M \cong \mathbb{R}^{n}$ and hence $m=1$. In this case, we can assume that $g=$ $g_{i j} d x^{i} d x^{j}$ is a Riemannian metric on $\mathbb{R}^{n}$ such that

$$
g_{i j}-\delta_{i j}, g^{i j}-\delta^{i j} \in H_{\delta}^{k}
$$

where $g^{i j}$ are the components of the inverse metric, satisfying $g^{i j} g_{j k}=\delta_{k}^{i}$.

\section{Local existence, uniqueness, and continuation}

We now state a local existence result for Ricci flow on asymptotically flat manifolds. For a proof, see [19], Section 3. Alternatively, one can use [21] to obtain local existence and then a separate argument for the preservation of the asymptotics $[6,15]$.

Theorem 3.1. Let $\hat{g}$ be an asymptotically flat metric of class $H_{\delta}^{k}$ with $\delta<0$ and $k>n / 2+3$. Then there exists a $T>0$ and a family $\{g(t), t \in[0, T)\}$ of asymptotically flat metrics of class $H_{\delta}^{k-2}$ such that $g(0)=\hat{g}$,

$$
g_{i j}-\delta_{i j}, g^{i j}-\delta^{i j} \in C^{1}\left([0, T), H_{\delta}^{k-2}\right),
$$

and $\partial_{t} g_{i j}=-2 R_{i j}$ for all $t \in[0, T)$. Moreover, $g(t, x) \in C^{\infty}((0, T) \times M)$ and $g_{i j}-\delta_{i j}, g^{i j}-\delta^{i j} \in C^{1}\left(\left[T_{1}, T_{2}\right], H_{\delta}^{\ell}\right)$ for any $\ell \geq 0$ and $0<T_{1}<T_{2}<T$. 
The following corollary is a straightforward consequence of the above local existence theorem, the weighted Sobolev inequality [2, Theorem 2.1], and the weighted multiplication lemma [16, Lemma 3.3]. See [19, Section 3], for details.

Corollary 3.2. Let $k>n / 2+4$ and $g(t)$ be the Ricci flow solution from Theorem 3.1. Then $R_{i j} \in C^{1}\left([0, T), H_{\delta-2}^{k-4}\right)$ and $g_{i j}(t)=\hat{g}_{i j}+f_{i j}(t)$, where $f_{i j} \in C^{1}\left([0, T), H_{\delta-2}^{k-4}\right)$. Moreover, if $k>n / 2+6, \delta<4-n$ and $\hat{R} \in L^{1}$ then $R(t) \in C^{1}\left([0, T), L^{1}\right)$.

Remark 3.3. In [2] Proposition 4.1, it is established that the mass of an asymptotically flat metric $g$ of class $H_{\delta}^{k} \subset W_{\delta}^{2,2 n /(n-2)}(k \geq 3)$ is well defined and given by the formula

$$
\operatorname{mass}(g):=\int_{S_{\infty}}\left(\partial_{j} g_{i j}-\partial_{i} g_{j j}\right) d S^{i}
$$

provided $\delta \leq(2-n) / 2$ and the Ricci scalar is both non-negative and integrable. So, by the above corollary, an initial asymptotically flat metric $\hat{g}$ of class $H_{\delta}^{k}$, where $k>n / 2+6$ and $\delta<\min \{4-n,(2-n) / 2\}$, with nonnegative and integrable Ricci scalar will yield a flow $g(t)$ for which the Ricci scalar continues to be non-negative and integrable for every $t>0$. Thus the mass of $g(t)$ remains well defined. Furthermore, since $g_{i j}-\hat{g}_{i j} \in H_{\delta-2}^{k-4} \subset$ $W_{\delta-2}^{1, \infty} \subset W_{2-n}^{1, \infty}$, it follows easily from the definition of the mass that

$$
\operatorname{mass}(g(t))=\operatorname{mass}(\hat{g}) \quad \text { for all } t \geq 0 .
$$

This result has also been established in [6] using different methods.

On asymptotically flat manifolds, the standard arguments for uniqueness and continuation of Ricci flow on compact manifolds carry over with only minor modifications. Again, see [19, Section 3] for details.

Theorem 3.4. Suppose $k>n / 2+4, \delta<0$ and $\hat{g}$ is an asymptotically flat metric of class $H_{\delta}^{k}$. Then Ricci flow $\partial_{t} g_{i j}=-2 R_{i j}$ with the initial condition $g(0)=\hat{g}$ has a unique solution on a maximal time interval $0 \leq t<T_{M} \leq \infty$. If $T_{M}<\infty$ then

$$
\limsup _{t \rightarrow T_{M}} \sup _{x \in \mathbb{R}^{n}}|\operatorname{Rm}(t, x)|_{g(t, x)}=\infty
$$


Rotationally symmetric Ricci flow on asymptotically flat manifolds 543

Moreover, for any $T \in\left[0, T_{M}\right), K=\sup _{0 \leq t \leq T} \sup _{x \in \mathbb{R}^{n}}|\operatorname{Rm}(t, x)|_{g(t, x)}<\infty$ and

$$
e^{-2 K T} \hat{g} \leq g(t) \leq e^{2 K T} \hat{g} \text { for all } t \in[0, T] .
$$

This theorem can also be proved by combining the uniqueness result in [4] with the local existence result in [21].

\section{Rotational symmetry}

\subsection{The coordinate system}

We now restrict our attention to flows evolving from a fixed initial metric that (i) is rotationally symmetric and admits no minimal hyperspheres, and (ii) is asymptotically flat of class $H_{\delta}^{k}$ with $\delta<0$ and $k>(n / 2)+4$. In an attempt to manage the several constants that will appear from here onward, we will sometimes use the notation $C_{x}^{+}$to denote a constant that bounds a quantity $x$ from above; dually, $C_{x}^{-}$will sometimes be used to denote a constant that bounds $x$ from below.

\section{Remark 4.1.}

(i) By Theorem 3.1, there exists a solution $\bar{g}(t)$ to Ricci flow satisfying

$$
\begin{aligned}
\bar{g}_{i j}-\delta_{i j}, \bar{g}^{i j}-\delta^{i j} & \in C^{1}\left(\left[0, T_{M}\right), H_{\delta}^{k-2}\right), \\
\bar{g}(t, x) & \in C^{\infty}\left(\left(0, T_{M}\right) \times \mathbb{R}^{n}\right), \quad \text { and } \bar{g}(0)=\hat{g} .
\end{aligned}
$$

(ii) From (4.1) and the weighted Sobolev embedding (see, [2, Theorem 1.2]), it follows that $\bar{g}(t) \in C^{1}\left(\left[0, T_{M}\right), C_{\delta}^{2}\right)$ and hence there exists a time dependent constant $C(t)$ such that

$$
\left|D_{x}^{I} \bar{g}_{i j}(t, x)\right| \leq \frac{C(t)}{\left(1+|x|^{2}\right)^{(|\delta|+|I|) / 2}}
$$

for all $(t, x) \in\left[0, T_{M}\right) \times \mathbb{R}^{n}$, and $|I| \leq 2$.

(iii) Since Ricci flow preserves isometries, each metric $g(t)$ is rotationally symmetric and hence

$$
\bar{g}(t, x)=q^{2}(t, r) d r^{2}+h^{2}(t, r) g_{\text {can }}
$$


for functions $q(t, r)$ and $h(t, r)$ which are $C^{1}$ in $t, C^{2}$ in $r, C^{\infty}$ in $t$ and $r$ for $t>0$, and satisfy

$$
\begin{gathered}
q(0, r)=a(r), \quad h(0, r)=r, \\
\left|\partial_{r}^{s}\left(q^{2}(t, r)-1\right)\right| \leq \frac{C(t)}{(1+r)^{|\delta|+s}} s=0,1,2, \\
\left|\partial_{r}^{s}\left(r^{-2} h^{2}(t, r)-1\right)\right| \leq \frac{C(t)}{(1+r)^{|\delta|+s}} s=0,1,2 .
\end{gathered}
$$

Since $\partial_{r} h(0, r)=\partial_{r} r=1$, it follows that there exist constants $0<C_{\partial_{r} h}^{-} \leq 1, C_{\partial_{r} h}^{+} \geq 1$, such that

$$
0<C_{\partial_{r} h}^{-} \leq \partial_{r} h(t, r) \leq C_{\partial_{r} h}^{+} \text {for all }(t, r) \in[0, T] \times(0, \infty)
$$

for some $T>0$. Note that $T$ has no a priori relation to $T_{M}$, the maximal existence time of the flowing metric (4.3). However, let $\tilde{T}$ be the largest time such that (4.7) holds whenever $T<\tilde{T}$. We will show in Section 4.3 that we can take $\tilde{T}=T_{M}$.

Letting $\left(\theta^{A}\right)$ denote angular coordinates on the sphere $\mathbb{S}^{n-1}$, the map

$$
\psi_{t}\left(r, \theta^{A}\right)=\left(h(t, r), \theta^{A}\right)
$$

defines a $C^{2}$ diffeomorphism on $\mathbb{R}^{n}$ for each $t \in[0, \tilde{T})$ which is smooth for all $t>0$. So then

$$
\psi_{t}^{-1}\left(r, \theta^{A}\right)=\left(\rho(t, r), \theta^{A}\right)
$$

for a function $\rho(t, r)$ that is $C^{1}$ in $t, C^{2}$ in $r, C^{\infty}$ in $r$ and $t$ for $t>0$, and satisfies

$$
h(t, \rho(t, r))=r, \quad \rho(t, h(t, r))=r, \quad \text { and } \quad \rho(0, r)=r
$$

for all $(t, r) \in[0, T] \times(0, \infty)$. Next, define

$$
g(t):=\left(\psi_{t}^{-1}\right)^{*} \bar{g}(t)
$$

Then we finally obtain that

$$
g(t)=f^{2}(t, r) d r^{2}+r^{2} g_{\mathrm{can}},
$$


Rotationally symmetric Ricci flow on asymptotically flat manifolds 545

where

$$
f(t, r)=\frac{q(t, \rho(t, r))}{\partial_{r} h(t, \rho(t, r))} \text { for all }(t, r) \in[0, \tilde{T}) \times(0, \infty)
$$

Note that $f(t, r)$ is $C^{1}$ in $t$, is $C^{2}$ in $r$, and $C^{\infty}$ in $r$ and $t$ for $t>0$. As well,

$$
\lim _{r \rightarrow \infty} f^{2}(t, r)=1
$$

(proof: from (4.5) we have $q^{2} \rightarrow 1$ and from (4.6) it is easy to check that $\partial_{r} h \rightarrow 1$; then apply these in $\left.(4.13)\right)$. Finally note that the mean curvature of constant- $r$ hyperspheres is

$$
H=\frac{1}{r f}
$$

so a minimal hypersphere occurs iff $f$ diverges at finite $r$ and some $t \in[0, \tilde{T}]$. We show in the following subsection that such a divergence cannot develop.

\subsection{Ricci flow in area radius coordinates}

The metric (4.12) is a solution of the Hamilton-DeTurck flow (1.2), at least for $t \in[0, \tilde{T})$. Now from (4.12) we can directly compute the Ricci curvature and obtain

(4.16) $\mathrm{Ric}=\frac{(n-1)}{r f(t, r)} \frac{\partial f}{\partial r} d r^{2}+\left[(n-2)\left(1-\frac{1}{f^{2}(t, r)}\right)+\frac{r}{f^{3}(t, r)} \frac{\partial f}{\partial r}\right] g_{\mathrm{can}}$.

We can then use the components of the flow equation (1.2) normal to $\partial / \partial r$ to determine $\xi$, expressed as a 1 -form, to be $\xi=\xi_{1}(t, r) d r$, where

$$
\xi_{1}=\left[\frac{(n-2)}{r}\left(f^{2}(t, r)-1\right)+\frac{(\partial f / \partial r)}{f(t, r)}\right] .
$$

We can then write the $r r$-component of (1.2) as a differential equation for $f$ and use (4.17) to eliminate $\xi$ from this equation. The result is

$$
\begin{aligned}
\frac{\partial f}{\partial t}= & \frac{1}{f^{2}} \frac{\partial^{2} f}{\partial r^{2}}-\frac{2}{f^{3}}\left(\frac{\partial f}{\partial r}\right)^{2}+\left(\frac{(n-2)}{r}-\frac{1}{r f^{2}}\right) \frac{\partial f}{\partial r} \\
& -\frac{(n-2)}{r^{2} f}\left(f^{2}-1\right) .
\end{aligned}
$$


This is our master equation upon which our global existence proof is based. Obviously $f(t, r)=1$ (flat space) is a solution, as is $f=$ const $\neq 1$ when $n=2$ (flat cone) but not for $n>2$.

We will now prove that minimal hyperspheres cannot form along the flow if none are present initially. A variant of this argument will be employed several times over in Section 5. Our technique is to prescribe limits as $r \rightarrow \infty$ and as $r \rightarrow 0$ on $f(t, r)$ or, depending on the situation, an expression involving $f$ (and, in the next section, its radial derivative as well). These limits constitute time-dependent bounds on the behaviour of the geometry over the time interval $[0, \tilde{T})$. But if the flow exists subject to these limits, then maximum principles will give bounds expressed solely in terms of the initial conditions. The bounds are therefore uniform in time and independent of $\tilde{T}$.

To see how this works, express (4.18) in terms of the variable

$$
w(t, r):=f^{2}(t, r)-1
$$

Then, working from (4.18), we see that $w$ obeys

$$
\frac{\partial w}{\partial t}=\frac{1}{f^{2}} \frac{\partial^{2} w}{\partial r^{2}}-\frac{3}{2 f^{4}}\left[\frac{\partial w}{\partial r}\right]^{2}+\left[\frac{n-2}{r}-\frac{1}{r f^{2}}\right] \frac{\partial w}{\partial r}-\frac{2(n-2)}{r^{2}} w
$$

Since $f(t, r)$ solves (refer equation 4.18) and obeys $\lim _{r \rightarrow 0} f^{2}(t, r)=$ $1=\lim _{r \rightarrow \infty} f^{2}(t, r)$, the corresponding $w=f^{2}-1$ will solve (4.20) with $\lim _{r \rightarrow 0} w(t, r)=0=\lim _{r \rightarrow \infty} w(t, r)$.

Proposition 4.2. Suppose that $w(t, r)$ is a classical solution of (4.20) for $(t, r) \in[0, \tilde{T}) \times[0, \infty)=: \tilde{D}$ and that $\lim _{r \rightarrow 0} w(t, r)=0=\lim _{r \rightarrow \infty} w(t, r)$ for all $t \in[0, \tilde{T})$. Then there exist constants $C_{w}^{-} \leq 0$ and $C_{w}^{+} \geq 0$ such that $C_{w}^{-} \leq w(t, r) \leq C_{w}^{+}$for all $(t, r) \in \tilde{D}$

Proof. First choose positive constants $0<r_{1}<r_{2}$ and restrict the domain to $r \in\left[r_{1}, r_{2}\right]$. Let $T<\tilde{T}$. By the maximum principle, if the maximum of $w$ on $[0, T] \times\left[r_{1}, r_{2}\right]$ is positive, it must lie on the parabolic boundary $P$ (which consists of those points where either $t=0, r=r_{1}$, or $r=r_{2}$ ). But now take the limits $r_{1} \rightarrow 0$ and $r_{2} \rightarrow \infty$. By assumption, $w\left(t, r_{1}\right)$ and $w\left(t, r_{2}\right)$ tend to zero in these limits, so for $r_{1}$ small enough and $r_{2}$ large enough, the maximum, if it is positive, lies on the initial boundary $\{(t, r) \mid t=0\}$ (and since $w(0,0)=0$, even when the maximum is zero it is realized on the initial 
Rotationally symmetric Ricci flow on asymptotically flat manifolds 547 boundary). Finally, take $T \rightarrow \tilde{T}$. This proves

$$
C_{w}^{+}:=\max _{r \in[0, \infty)}\{w(0, r)\}=\max _{\tilde{D}}\{w(t, r)\} \geq 0 .
$$

Dually, by the minimum principle, if the minimum of $w$ on $[0, T] \times\left[r_{1}, r_{2}\right]$ is negative, it must lie on $P$, and the argument proceeds as before, yielding

$$
C_{w}^{-}:=\min _{r \in[0, \infty)}\{w(0, r)\}=\min _{\tilde{D}}\{w(t, r)\} \leq 0
$$

Corollary 4.3. Define constants $C_{f^{2}}^{ \pm}$such that $0<C_{f^{2}}^{-}:=\min _{r \in[0, \infty)}$ $\left\{a^{2}(r)\right\}$ and let $C_{f^{2}}^{+}:=\max _{r \in[0, \infty)}\left\{a^{2}(r)\right\}(a(r)$ is defined in (4.4)). Then

$$
0<C_{f^{2}}^{-} \leq f^{2}(t, r) \leq C_{f^{2}}^{+} .
$$

for all $(t, r) \in \tilde{D}=[0, \tilde{T}) \times[0, \infty)$.

Proof. Using $w:=f^{2}-1$ and noting in particular that $w(0, r)=f^{2}(0, r)-$ $1=a^{2}(r)-1$, apply Proposition 4.2 and use $C_{w}^{ \pm}+1=C_{f^{2}}^{ \pm}$.

Now we say that a minimal hypersphere forms along the flow iff $f(t, r)$ diverges in $\tilde{D}=[0, \tilde{T}) \times[0, \infty)$.

Corollary 4.4. If no minimal sphere is present initially then none forms.

Proof. From Corollary 4.3, the classical solutions $f$ of (4.18) developing from initial data (4.1) are bounded uniformly in $t$ on $[0, \tilde{T})$.

\subsection{The continuation principle in area radius coordinates}

To adapt the continuation principle of Section 3.2 to the rotationally symmetric case, we must deal with the following point. While we can assume the solution of Ricci flow in the coordinate system (4.3) to exist for all $t<T_{M}$, the diffeomorphism transforming the coordinates to those of (4.12) is, so far, only defined for $t<\tilde{T}$, and perhaps $\tilde{T}<T_{M}$.

Proposition 4.5. $\tilde{T}=T_{M}$.

Proof. Let $K=\sup _{0 \leq t \leq T^{\prime}}\|\mathrm{Rm}\|_{L^{\infty}}$. But $\bar{R}_{i j k l}$ is bounded on $\left[0, T^{\prime}\right]$ (indeed, on any closed subinterval of $\left.\left[0, T_{M}\right)\right)$, so we can use (3.4), which states that 
for all $(t, r) \in\left[0, T^{\prime}\right] \times[0, \infty)$

$$
e^{-2 K T^{\prime}} C_{f^{2}}^{-} \leq e^{-2 K T^{\prime}} a^{2}(r) \leq q^{2}(t, r) \leq e^{2 K T^{\prime}} a^{2}(r) \leq e^{2 K T^{\prime}} C_{f^{2}}^{+} .
$$

Here the inner two inequalities come from (3.4) and the outer two are just the definitions of the constants $C_{f^{2}}^{ \pm}$.

Assume by way of contradiction that $\tilde{T}<T_{M}$. If we restrict attention to $t \in[0, \tilde{T})$ then we can divide $(4.24)$ by $(4.23)$. This yields

$$
0<e^{-2 K T^{\prime}} \frac{C_{f^{2}}^{-}}{C_{f^{2}}^{+}} \leq \frac{q^{2}(t, r)}{f^{2}(t, r)} \leq e^{2 K T^{\prime}} \frac{C_{f^{2}}^{+}}{C_{f^{2}}^{-}}
$$

on $[0, \tilde{T})$. Using $(4.13)$, we can rewrite this as

$$
0<e^{-2 K T^{\prime}} \frac{C_{f^{2}}^{-}}{C_{f^{2}}^{+}} \leq \frac{\partial h}{\partial r} \leq e^{2 K T^{\prime}} \frac{C_{f^{2}}^{+}}{C_{f^{2}}^{-}}
$$

on $[0, \tilde{T})$. We see by comparison of this to (4.7) that the constants that appear in (4.7) are independent of $T$. But the $\leq$ signs give closed relations so, by relaxing the constant bounds slightly if necessary (keeping the lower bound positive of course), we can extend (4.26) (equivalently, (4.7)) to $[0, \tilde{T}]$ and then to some interval $\left[0, T^{\prime}\right) \supset[0, \tilde{T}]$. This contradicts the assumption that $\tilde{T}<T_{M}$, and since necessarily $\tilde{T} \leq T_{M}$ we must therefore conclude that $\tilde{T}=T_{M}$.

Thus the diffeomorphism $(4.8-4.11)$ is defined for all $t \in\left[0, T_{M}\right)$. The square of the norm of the curvature tensor is given by

$$
|\mathrm{Rm}|^{2}=R_{i j k l} R^{i j k l}=2(n-1) \lambda_{1}^{2}+(n-1)(n-2) \lambda_{2}^{2},
$$

where

$$
\lambda_{1}(t, r)=\frac{1}{r f^{3}(t, r)} \frac{\partial f(t, r)}{\partial r}
$$

and

$$
\lambda_{2}(t, r)=\frac{1}{r^{2}}\left(1-\frac{1}{f^{2}(t, r)}\right)
$$

are the sectional curvatures in planes containing and orthogonal to $d r$, respectively. Now in terms of the curvature tensor $\bar{R}_{i j k l}$ of $\bar{g}(t)$ we have that

$$
|\mathrm{Rm}|=|\overline{\mathrm{Rm}}| \circ \psi_{t}^{-1}
$$


Rotationally symmetric Ricci flow on asymptotically flat manifolds 549

But $\bar{R}_{i j k l}$ is bounded on any interval $\left[0, T^{\prime}\right]$ with $T^{\prime}<T_{M}$ and thus the sectional curvatures are bounded functions of $(t, r) \in\left[0, T^{\prime}\right] \times[0, \infty)$, using Proposition 4.5. Thus

$$
\begin{aligned}
& C_{\lambda_{1}}^{-}(t) \leq \lambda_{1}(t, r)=\frac{1}{r f^{3}} \frac{\partial f}{\partial r} \leq C_{\lambda_{1}}^{+}(t), \\
& C_{\lambda_{2}}^{-}(t) \leq \lambda_{2}(t, r)=\frac{1}{r^{2}}\left(1-\frac{1}{f^{2}(t, r)}\right) \leq C_{\lambda_{2}}^{+}(t),
\end{aligned}
$$

for all $t \in\left[0, T_{M}\right)$. In particular, the limits $r \rightarrow 0$ of these quantities exist at each fixed $t$. It also follows easily from the fall-offs (4.5) and (4.6) that

$$
\lim _{r \rightarrow \infty} r^{-|\delta|-s} \partial_{r}\left(f^{2}(t, r)-1\right)=0
$$

for $s=0,1,2$ and all $t \in\left[0, T^{\prime}\right)$. Thus we have that

Proposition 4.6. The function $f(t, r)$ given by (4.13) solves the PDE (4.18) on the region $\left[0, T_{M}\right) \times(0, \infty)$, equals a(r) at time $t=0$, and satisfies the boundary conditions

$$
\lim _{r \rightarrow 0} \frac{1-f^{2}(t, r)}{r^{2}}=L_{1}(t), \quad \lim _{r \rightarrow 0} \frac{\partial_{r} f(t, r)}{r}=L_{2}(t),
$$

for locally bounded functions $L_{1}, L_{2}:\left[0, T_{M}\right) \rightarrow \mathbb{R}$ and

$$
\lim _{r \rightarrow \infty} r^{-|\delta|-s} \partial_{r}\left(f^{2}(t, r)-1\right)=0 \quad(s=0,1,2)
$$

for all $t \in\left[0, T_{M}\right)$ and $\delta<0$.

Proof. To obtain the boundary conditions (4.34), multiply (4.31) by $f^{3}$, (4.32) by $f^{2}$, take the limit, and use that $f$ is a bounded function of $r$. The fact that $f$ solves (4.18), subject to these conditions, for all $(t, r) \in$ $\left[0, T_{M}\right) \times[0, \infty)$ follows from the facts that (i) $q$ and $h$ enter (4.3) which solves Ricci flow (1.1), (ii) $f$ enters (4.12) which solves Hamilton-DeTurck flow (1.2), and (iii) the diffeomorphism (4.8-4.11) relating these flows is valid for all such $(t, r)$ (Proposition 4.5).

Theorem 4.7. If there exists a constant $C_{\lambda}>0$ independent of $T_{M}$ such that

$$
\sup _{0<r<\infty}\left(\left|\lambda_{1}(t, r)\right|+\left|\lambda_{2}(t, r)\right|\right) \leq C_{\lambda},
$$

then $T_{M}=\infty$. 
Proof. From Proposition 4.6, the solution $f$ of (4.18) exists up to time $T_{M}$. From (4.27-4.30), if the sectional curvatures $\lambda_{1}$ and $\lambda_{2}$ are bounded independent of $T_{M}$, then so is $|\overline{\mathrm{Rm}}|^{2}$, and then by Theorem 3.5 we have $T_{M}=\infty$.

\subsection{Quasi-local mass}

This subsection is a brief aside, not necessary for our main results, but intended to relate our results to the motivation discussion in the introduction.

One of the more popular quasi-local mass formulations is the Brown-York mass. The Brown-York quasi-local mass contained within a closed hypersurface $\Sigma$ is defined to be

$$
\mu[\Sigma]:=\int_{\Sigma}\left(H_{0}-H\right) d \Sigma
$$

where $H$ is the mean curvature of $\Sigma$ and $H_{0}$ is the mean curvature of the image of $\Sigma$ under an isometric embedding of $\Sigma$ into flat space (assuming there is such an embedding). In the case of a hypersphere $r=b(t)$, whose coordinate radius we will allow to possibly change in time, we have (using (4.15) and writing $d \Omega$ to represent the canonical volume element on the $(n-1)$-sphere)

$$
\begin{aligned}
\mu(t) & =\int_{\mathbb{S}^{n-1}} \frac{1}{b(t)}\left(1-\frac{1}{f(t, b(t))}\right) b^{n-1}(t) d \Omega \\
& =b^{n-2}(t)\left(1-\frac{1}{f(t, b(t))}\right) \operatorname{vol}\left(\mathbb{S}^{n-1}, \text { can }\right) .
\end{aligned}
$$

Comparing to (4.32), we can relate quasi-local mass to sectional curvature by

$$
\frac{1}{b^{n}(t)}\left(1+\frac{1}{f(t, b(t))}\right) \mu(t, b(t))=\lambda_{2}(t, b(t)) \operatorname{vol}\left(\mathbb{S}^{n-1}, \text { can }\right) .
$$

Proposition 4.8. The sign of the Brown-York quasi-local mass within the hypersphere $r=b(t)$ at time $t$ is determined by the sign of $\lambda_{2}(t, b(t))$, and

$$
\lim _{t \rightarrow \infty} \lambda_{2}(t, b(t))=0 \Longleftrightarrow \lim _{t \rightarrow \infty} \mu(t, b(t))=0
$$

Proof. Obvious from (4.39) and (4.23). 
Rotationally symmetric Ricci flow on asymptotically flat manifolds 551

Perhaps the three most interesting kinds of hyperspheres are those of

(i) fixed surface area

$$
b(t)=b_{0}=\text { const }>0,
$$

(ii) fixed volume contained within

$$
\int_{0}^{b(t)} \int_{\mathbb{S}^{n-1}} f(t, r) r^{n-1} d r d \Omega=: V_{0}=\text { const }>0, \quad \text { and }
$$

(iii) fixed proper radius

$$
\int_{0}^{b(t)} f(t, r) d r=: R_{0}=\text { const }>0 .
$$

In either case, it is easy to see that

$$
0<C_{b}^{-} \leq b(t) \leq C_{b}^{+},
$$

where obviously $C_{b}^{ \pm}=b_{0}$ for the fixed area case, while

$$
C_{b}^{ \pm}=\frac{n V_{0}}{C_{f^{2}}^{\mp} \operatorname{vol}\left(\mathbb{S}^{n-1}, \mathrm{can}\right)}
$$

for the fixed volume case and

$$
C_{b}^{ \pm}:=\frac{R_{0}}{C_{f^{2}}^{\mp}}
$$

for the fixed proper radius case.

Remark 4.9. In Section 5.1, we prove that $\lambda_{2}(t, r) \sim 1 / t$ for large $t$ and fixed $r$. Thus, for all three kinds of hyperspheres discussed above, the quasilocal mass vanishes like $1 / t$ as $t \rightarrow \infty$.

\section{Immortality and convergence}

In the next two subsections we show that the sectional curvatures $\lambda_{1}$ and $\lambda_{2}$ are bounded on $t \in\left[0, T_{M}\right.$ ). (Equivalently, we obtain bounds on the quasilocal mass and its radial derivative.) This permits us to invoke Theorem 4.7 to conclude that the solution is immortal. In fact, we find bounds that actually decay in time, going to zero in the limit $t \rightarrow \infty$. This implies that the flow converges in the limit to a space with vanishing sectional curvatures; 
i.e., to a flat space. In Section 5.3, we prove that it converges to Euclidean space $\mathbb{E}^{n}$.

In this section, we use $T$ to denote an arbitrary time that is less than the maximal time of existence, i.e., $0<T<T_{M}$.

\subsection{The decay of $\lambda_{2}$}

Short-time existence guarantees that $f^{2}(t, r)-1 \in \mathcal{O}\left(r^{2}\right)$ as $r \rightarrow 0$. Specifically, for all $r<r_{0}$ and for $0 \leq t<T_{M}$, there is a function $C(t)$ such that

$$
|w(t, r)|=\left|f^{2}(t, r)-1\right|<C(t) r^{2} .
$$

This follows by applying the boundedness of $f^{2}$ (4.23) to equation (4.32) governing $\lambda_{2}$, which can be written (by choosing $C(t)$ less than optimally, perhaps) as

$$
r^{2}\left|\lambda_{2}(t, r)\right|=\left|\frac{1}{f^{2}}-1\right|<C(t) r^{2}
$$

To apply the continuation principle, we need to prove that $C(t)$ is bounded in $t$. In this section we will prove more: we will show that $C(t)$ can be taken to decay in time, converging to zero in the limit $t \rightarrow \infty$, so that the sectional curvature $\lambda_{2}$ decays to zero as well.

If $w=f^{2}-1$ decays, then, based on the parabolic form of (4.20), one might speculate that this decay would go roughly like $r^{2} / t$, or inverse "parabolic time". If so, then the function $g(t, r)\left(f^{2}-1\right)$ should be bounded if we take $g \sim t / r^{2}$. We will show below that this expectation is basically correct.

We do not take $g=t / r^{2}$ exactly. For small $t$, we will modify the form $g \sim t / r^{2}$ so that $g$ does not vanish at $t=0$. For small $r$, the form $g \sim t / r^{2}$ is problematical because we cannot specify a priori the behaviour of $\left(1 / r^{2}\right)\left(f^{2}-1\right)$ on approach to $r=0$. This behaviour is governed by $C(t)$, the very quantity we seek to control as the outcome of the argument, so we cannot specify it as input. We therefore choose instead small $r$ behaviour of the form $g(t, r) \sim 1 / r^{m}, m<2$, and only later do we take $m \rightarrow 2$. For $m<2, g(t, r)\left(f^{2}-1\right)$ is very well controlled a priori for small $r$ : it goes to zero. Lastly, as foreshadowed by (5.2), we need to apply these considerations not only with $f^{2}-1$ but also to $\left(1 / f^{2}\right)-1$. The same heuristic reasoning leads us then to consider functions of the form $g(t, r)\left(\left(1 / f^{2}\right)-1\right)$ with the same $g(t, r)$. 
Rotationally symmetric Ricci flow on asymptotically flat manifolds 553

Definition 5.1. Let $f$ be defined by $(4.13)^{5}$ and therefore have all the properties outlined in Section 4. For such an $f$, define the $u_{m}$ functions, $m \in(0,2]$, on $\left[0, T_{M}\right) \times[0, \infty)$ by

$$
\begin{aligned}
& u_{m}(t, r):=\left(\frac{1+t}{r^{m}+r^{2}}\right)\left(\frac{1}{f^{2}(t, r)}-1\right) \text { for } r>0, \\
& u_{m}(t, 0):=\lim _{r \rightarrow 0} u_{m}(t, r) .
\end{aligned}
$$

The $u_{m}$ functions have the following properties, which follow from the flow equation (4.18) for $f$, Proposition 4.6, and equation (4.14).

(i) $u_{m}(t, 0)=0$ for all $0<m<2$ and $\lim _{r \rightarrow \infty} u_{m}(t, r)=0$ for all $0<m \leq 2$.

(ii) For fixed $t$ and $r \neq 0$, the map $m \mapsto u_{m}(t, r)$ is continuous at $m=2$.

$$
\lambda_{2}=-\frac{2}{1+t} u_{2} .
$$

(iv) The $u_{m}$ obey a maximum principle, as we will show below.

(v) By direct calculation starting from (4.18), the $u_{m}$ obey the differential equation:

$$
\begin{aligned}
\frac{\partial u_{m}}{\partial t}= & \frac{1}{f^{2}} \frac{\partial^{2} u_{m}}{\partial r^{2}}-\frac{\left(r^{m}+r^{2}\right)}{2(1+t)}\left(\frac{\partial u_{m}}{\partial r}\right)^{2}-\frac{\left(2 r+m r^{m-1}\right)}{(1+t)} u_{m} \frac{\partial u_{m}}{\partial r} \\
& +\left[\frac{2\left(2 r+m r^{m-1}\right)}{\left(r^{m}+r^{2}\right) f^{2}}-\frac{1}{r f^{2}}+\frac{(n-2)}{r}\right] \frac{\partial u_{m}}{\partial r} \\
& -\frac{(2-m)(m+n-2)}{r^{2}\left(1+r^{2-m}\right)} u_{m} \\
& +\frac{1}{(1+t)}\left\{\frac{1}{1+r^{2-m}}\left[u_{m}-((4-m)(m+n-2)+m(n-2)) u_{m}^{2}\right]\right. \\
& +\frac{r^{2-m}}{1+r^{2-m}}\left[u_{m}-2(n-1) u_{m}^{2}\right] \\
& \left.+\frac{r^{m-2}}{1+r^{2-m}}\left[(m-2)(m+n-2)-m\left(\frac{m}{2}+n-2\right)\right] u_{m}^{2}\right\} .
\end{aligned}
$$

${ }^{5}$ wherein, of course, $q$ and $h$ arise from an asymptotically flat Ricci flow of rotationally symmetric initial data obeying the conditions of Theorem 1.1. 
This PDE is the starting point for the maximum principle, which we now derive.

Proposition 5.2. For $u_{m}(t, r)$ defined by Definition 5.1, there is a constant $C_{u}^{+}$which depends only on the initial data $a(r)=f(0, r)$ such that $u_{m}(t, r) \leq$ $C_{u}^{+}$for all $t \in\left[0, T_{M}\right)$ and all $m \in(0,2)$.

Proof. The technique will be to solve (4.18) for $f$, given initial data obeying the bounds in Corollary 4.3. From this initial data, we can construct initial data for $u_{m}$ using from (5.3) that

$$
u_{m}(0, r):=\frac{1}{r^{m}+r^{2}}\left(\frac{1}{f^{2}(0, r)}-1\right)=-\frac{\lambda_{2}(0, r)}{1+r^{m-2}} .
$$

Now by the assumed differentiability and asymptotic flatness of the initial metric stated in Theorem 1.1, the initial sectional curvature $\lambda_{2}(0, r)$ is bounded. In particular, then by $(5.6) u_{m}(0, r)$ is bounded above on $r \in[0, \infty)$ by a constant $C_{u}^{+}$which depends only on the initial metric (thus on $a(r)$ as in (4.4)) and so does not depend on $m$. Without loss of generality, we choose $C_{u}^{+} \geq(1 / 2(n-1))$, for reasons that will become clear. Now it remains to be shown that $u_{m}(t, r)$ is bounded above for all time $t \geq 0$ by a bound that is dependent only on $u_{m}(0, r)$. Of course, the initial data $u_{m}(0, r)$ will vary with $m$ (because of the denominator of (5.6); but $f(0, r)=a(r)$ and, thus, $\lambda_{2}(0, r)$ are of course independent of $m$ ), but $C_{u}^{+}$will always provide an $m$-independent upper bound which will then bound the full solution.

First restrict consideration to the compact domain $D=[0, T] \times\left[r_{1}, r_{2}\right]$, $0<r_{1}<r_{2}, T<T_{M}$, with parabolic boundary $P$ (as defined in the proof of Proposition 4.2). Now consider in (5.5) the terms that do not contain derivatives. There are three such terms, each comprised of a function of $r$ multiplying a factor in square brackets. One can easily check (e.g., by direct substitution; keep in mind that $m \in(0,2)$ and $n \geq 3)$ that in (5.5) each of these factors in square brackets is negative whenever

$$
u_{m}>\frac{1}{2(n-1)}
$$

SO

$$
\begin{aligned}
\frac{\partial u_{m}}{\partial t}< & \frac{1}{f^{2}} \frac{\partial^{2} u_{m}}{\partial r^{2}}-\frac{\left(r^{m}+r^{2}\right)}{2(1+t)}\left(\frac{\partial u_{m}}{\partial r}\right)^{2}-\frac{\left(2 r+m r^{m-1}\right)}{(1+t)} u \frac{\partial u_{m}}{\partial r} \\
& +\left[\frac{2\left(2 r+m r^{m-1}\right)}{\left(r^{m}+r^{2}\right) f^{2}}-\frac{1}{r f^{2}}+\frac{(n-2)}{r}\right] \frac{\partial u_{m}}{\partial r}
\end{aligned}
$$


Rotationally symmetric Ricci flow on asymptotically flat manifolds 555

then. Applying the usual maximum principle argument to this inequality (i.e., evaluating both sides at a hypothesized local maximum and observing that the inequality cannot then be satisfied), we conclude that $u_{m}$ has no maximum greater than $(1 / 2(n-1))$ in $D \backslash P$.

By the properties of $u_{m}$ listed above, we have $u_{m}(t, r) \rightarrow 0$ both for $r \rightarrow 0$ and for $r \rightarrow \infty$. Thus, as with the proof of Proposition 4.2, if the maximum of $u_{m}$ is $>(1 / 2(n-1))$ (or merely positive) and lies on the parabolic boundary with $r_{1}$ chosen small enough and $r_{2}$ large enough, it must lie on the initial boundary. Taking the limits $r_{1} \rightarrow 0$ and $r_{2} \rightarrow \infty$, then we see that

$$
u_{m}(t, r) \leq \max \left\{\frac{1}{2(n-1)}, \sup _{r \in[0, \infty)}\left\{u_{m}(0, r)\right\}\right\} \leq C_{u}^{+},
$$

for all $(t, r) \in[0, T] \times[0, \infty)$. But this holds for any $T<T_{M}$, so it holds for $(t, r) \in\left[0, T_{M}\right) \times[0, \infty)$.

Corollary 5.3. Proposition 5.2 extends to the case $m=2$ and yields

$$
\lambda_{2}(t, r) \geq-\frac{2 C_{u}^{+}}{1+t}=: \frac{C_{\lambda_{2}}^{-}}{1+t} .
$$

Proof. As in Proposition 5.2, we solve (4.18) with the assumed initial data to find $f$, from which we construct $u_{m}$ for, say, $0<m \leq 2$. Fixing any $t \in$ $\left[0, T_{M}\right)$ and any $r \neq 0$, the map $m \mapsto u_{m}(t, r)=\left((1+t) / r^{2}+r^{m}\right)$ $\left(\left(1 / f^{2}(t, r)\right)-1\right)$ is obviously continuous at $m=2$. This and Proposition 5.2 imply that $u_{2}(t, r) \leq C_{u}^{+}$for all $r>0$. By the continuity of $r \rightarrow u_{2}(t, r)$, then $u_{2}(t, 0) \leq C_{u}^{+}$as well, for all $t \in\left[0, T_{M}\right)$. Now use (5.4).

Thus $\lambda_{2}$ is bounded below by a bound that tends to zero in the limit of long times. Next we need a similarly decaying bound from above. To get it, we work with the following class of functions:

Definition 5.4. Let $f$ be defined by (4.13) and therefore have all the properties outlined in Section 4. For such an $f$, the $v_{m}$ functions, $m \in(0,2]$ are defined on $\left[0, T_{M}\right) \times[0, \infty)$ as

$$
\begin{aligned}
& v_{m}(t, r):=\left(\frac{1+t}{r^{m}+r^{2}}\right)\left(f^{2}(t, r)-1\right) \text { for } r>0, \\
& v_{m}(t, 0):=\lim _{r \rightarrow 0} v_{m}(t, r) .
\end{aligned}
$$


These functions have essentially the same properties as those listed for the $u_{m}$, but the relation to $\lambda_{2}$ is now

$$
v_{2}(t, r)=\frac{1}{2}(1+t) f^{2}(t, r) \lambda_{2}(t, r)
$$

and the $v_{m}$ obey the PDE [computed directly from (4.18) and (5.11)]

$$
\begin{aligned}
\frac{\partial v_{m}}{\partial t}= & \frac{1}{f^{2}} \frac{\partial^{2} v_{m}}{\partial r^{2}}-\frac{3\left(r^{m}+r^{2}\right)}{2 f^{4}(1+t)}\left(\frac{\partial v_{m}}{\partial r}\right)^{2} \\
& +\left[\frac{2\left(m r^{m-1}+2 r\right)}{\left(r^{m}+r^{2}\right) f^{2}}-\frac{3\left(m r^{m-1}+2 r\right)}{(1+t) f^{4}}+\frac{n-2}{r}-\frac{1}{r f^{2}}\right] \frac{\partial v_{m}}{\partial r} \\
& +\left[1-\frac{3\left(m r^{m-1}+2 r\right)^{2}}{2\left(r^{m}+r^{2}\right) f^{4}} v_{m}\right] \frac{v_{m}}{1+t} \\
& +\frac{(m-2)}{r^{2}}\left(\frac{r^{m}}{r^{m}+r^{2}}\right)\left(n-2+\frac{m}{f^{2}}\right) v_{m}
\end{aligned}
$$

We must of course prove that the $v_{m}$ obey a maximum principle. In fact, Proposition 5.2 holds with $v_{m}$ replacing $u_{m}$ and with $m$ restricted this time to $1<m<2$. Just as with Corollary 5.3, the result can be extended to cover $m=2$. To prove this, it will help to note that when $v_{m} \geq 0, n \geq 3$, and $1<m<2$, then we can discard most of the nonderivative terms in (5.13) to obtain

$$
\begin{aligned}
\frac{\partial v_{m}}{\partial t} \leq & \frac{1}{f^{2}} \frac{\partial^{2} v_{m}}{\partial r^{2}}-\frac{3\left(r^{m}+r^{2}\right)}{2 f^{4}(1+t)}\left(\frac{\partial v_{m}}{\partial r}\right)^{2} \\
& +\left[\frac{2\left(m r^{m-1}+2 r\right)}{\left(r^{m}+r^{2}\right) f^{2}}-\frac{3\left(m r^{m-1}+2 r\right)}{(1+t) f^{4}}+\frac{n-2}{r}-\frac{1}{r f^{2}}\right] \frac{\partial v_{m}}{\partial r} \\
& +\frac{v_{m}}{(1+t)}\left[1-\frac{6 v_{m}}{f^{4}}\right], v_{m}>0
\end{aligned}
$$

Proposition 5.5. There is a constant $C_{v}^{+}$which depends only on the initial data $f(0, r)=a(r)$ such that $v_{m}(t, r)<C_{v}^{+}$for all $(t, r) \in\left[0, T_{M}\right) \times[0, \infty)$ and all $m \in(1,2)$.

Proof. The proof follows that of Proposition 5.2. Consider first the initial data

$$
v_{m}(0, r)=\left(\frac{1}{r^{m}+r^{2}}\right)\left(f^{2}(0, r)-1\right)=\left(\frac{1}{1+r^{m-2}}\right) \frac{w(0, r)}{r^{2}} \leq C_{v}^{+}
$$


Rotationally symmetric Ricci flow on asymptotically flat manifolds 557

because $\left(|w(0, r)| / r^{2}\right)$ is bounded, where $C_{v}^{+}$is independent of $m$. This time, we will choose without loss of generality that $C_{v}^{+} \geq(1 / 6)\left(C_{f^{2}}^{+}\right)^{2}$, for reasons that will become clear below.

Again we work first on the domain $D=[0, T] \times\left[r_{1}, r_{2}\right], 0<r_{1}<r_{2}$, with parabolic boundary $P$. Observe that if $v_{m}>(1 / 6)\left(C_{f^{2}}^{+}\right)^{2}$, the last term in (5.14) will be negative. As before, elementary arguments applied to (5.14) imply that this term cannot be negative at a maximum in $D \backslash P$, and thus such a maximum can occur only on $P$. Also as before, we take $r_{1} \rightarrow 0$, $r_{2} \rightarrow \infty$ and since $v_{m}$ vanishes in both limits, the maximum of $v_{m}$, if it is greater than $(1 / 6)\left(C_{f^{2}}^{+}\right)^{2}$, must occur on the initial boundary where $t=0$. Thus we obtain for any $(t, r) \in[0, T] \times[0, \infty)$ that

$$
v_{m}(t, r) \leq \max \left\{\frac{1}{6}\left(C_{f^{2}}^{+}\right)^{2}, \max _{r \in[0, \infty)}\left\{v_{m}(0, r)\right\}\right\} \leq C_{v}^{+},
$$

and $C_{v}^{+}$does not depend on $m$. It also does not depend on $T$ and so the result extends to hold for all $(t, r) \in\left[0, T_{M}\right) \times[0, \infty)$.

Remark 5.6. For use in the next subsection, we observe that in virtue of this result $u_{m}$ is now bounded below, as well as above, on $(t, r) \in\left[0, T_{M}\right) \times$ $[0, \infty)$ by a bound that depends only on the initial data for $f$ and so is independent of $m$. The proof is to observe that $u_{m}=-v_{m} / f^{2} \geq-C_{v}^{+} / C_{f^{2}}^{-}=$: $C_{u}^{-}$. We define

$$
C_{u}:=\max \left\{\left|C_{u}^{ \pm}\right|\right\}
$$

which bounds the magnitude of $\left|u_{m}\right|$ and is independent of $m$.

Corollary 5.7. Proposition 5.5 extends to the case $m=2$ and yields

$$
\lambda_{2}(t, r) \leq \frac{2 C_{v}^{+}}{C_{f^{2}}^{-}(1+t)}=: \frac{C_{\lambda_{2}}^{+}}{1+t} .
$$

Proof. The extension to $m=2$ follows exactly as in Corollary 5.3. Equation (5.18) follows directly from (5.12).

Proposition 5.8. $\left|\lambda_{2}\right|$ is bounded on $\left[0, T_{M}\right) \times[0, \infty)$ and if $T_{M}=\infty$ then $\lambda_{2}$ converges uniformly to zero as $t \rightarrow \infty$.

Proof. Immediate from Corollaries 5.3 and 5.7.

In this regard, note that by Theorem 4.7 we can assume $T_{M}=\infty$ if we can bound $\lambda_{1}$, which we now proceed to do. 


\subsection{The decay of $\lambda_{1}$}

A lower bound and decay estimate on $\lambda_{1}$ is now easy to obtain. It is quickest to work from the flow equation for the scalar curvature, which is

$$
\begin{aligned}
\frac{\partial R}{\partial t} & =\Delta R+\xi \cdot \nabla R+2 R_{i j} R^{i j} \\
& \geq \Delta R+\xi \cdot \nabla R+\frac{2}{n} R^{2},
\end{aligned}
$$

with $\xi=\xi_{1} d r$ given by (4.17) and where we used the elementary identity $R^{i j} R_{i j} \geq(1 / n) R^{2}$. Inequality (5.19) gives a well-known minimum principle for $R$. Moreover, if we define

$$
\tilde{R}:=(1+t) R,
$$

we obtain from (5.19) that

$$
\frac{\partial \tilde{R}}{\partial t} \geq \Delta \tilde{R}+\xi \cdot \nabla \tilde{R}+\frac{1}{(1+t)}\left(\frac{2}{n} \tilde{R}^{2}+\tilde{R}\right)
$$

which also has a minimum principle.

Proposition 5.9. If $R$ is the scalar curvature of a Ricci flow developing from asymptotically flat initial data on a manifold $M$ then there is a constant $C_{R}^{-} \leq 0$ such that on $\left[0, T_{M}\right) \times[0, \infty) \ni(t, r)$ we have

$$
R \geq \frac{C_{R}^{-}}{1+t}
$$

For notational convenience, we give the proof for the special case of interest, a rotationally symmetric flow on $\mathbb{R}^{n}$, but the proof clearly generalizes to arbitrary asymptotically flat flows.

Proof. First take $t \in[0, T], T<T_{m}$. Let $B_{0}(a)$ be the ball of coordinate radius $r=a$ about the origin $0 \in \mathbb{R}^{n}$ at time $t$. Applying elementary minimum principle arguments to (5.21), it is clear that either the minimum of $\tilde{R}$ in $[0, T] \times B_{0}(a)$ occurs on the parabolic boundary $P$ or $\tilde{R} \geq-(n / 2)$. Now the parabolic boundary has an initial component $t=0$ and a spatial component which is a sphere $r=a$ for all $t>0$. By asymptotic flatness, $R \rightarrow 0$ as $a \rightarrow \infty$ and hence $\tilde{R} \rightarrow 0$ as well. Taking this limit, we conclude that if $\tilde{R}$ is anywhere less than $-(n / 2)$, then the minimum of $\tilde{R}$ over all $(t, x) \in[0, T] \times \mathbb{R}^{n}$ exists and is realized on the initial boundary. 
Rotationally symmetric Ricci flow on asymptotically flat manifolds 559

Thus choose $C_{R}^{-}=\min \left\{-(n / 2), \inf _{r}\{R(0, r)\}\right\}$, which is obviously independent of $T$, so finally take $T \rightarrow T_{M}$. Then $\tilde{R} \geq C_{R}^{-} \Rightarrow R \geq C_{R}^{-}(1+t)$ for all $(t, r) \in\left[0, T_{M}\right) \times[0, \infty)$.

Corollary 5.10. Then $\lambda_{1}(t, r)$ is bounded below on $\left[0, T_{M}\right) \times[0, \infty) \ni$ $(t, r)$ by

$$
\lambda_{1}(t, r) \geq \frac{1}{(1+t)}\left(\frac{C_{R}^{-}}{2(n-1)}-\frac{(n-2) C_{v}^{+}}{C_{f^{2}}^{-}}\right)=: \frac{C_{\lambda_{1}}^{-}}{1+t} .
$$

Proof. This follows from the formula

$$
R=2(n-1) \lambda_{1}+(n-1)(n-2) \lambda_{2}
$$

for the scalar curvature in terms of the sectional curvatures, equation (5.22), and the upper bound (5.18) on $\lambda_{2}$.

Now we turn attention to finding an upper bound and decay estimate. We have to work harder than we did for the lower bound, but we can apply essentially the same strategy as we used to prove boundedness and convergence of $\lambda_{1}$. Once again, the main issue will be control of $\lambda_{1}$ at $r=0$, and we will be forced to work with a sequence of functions with known behaviour at $r=0$. This time, we have found that a choice well suited to our purpose is given by

Definition 5.11. Let $f$ be defined by (4.13) and therefore have all the properties outlined in Section 4. For such an $f$, define the $y_{m}$ functions, $m \in(1,2]$, on $\left[0, T_{M}\right) \times[0, \infty)$ by

$$
\begin{aligned}
& y_{m}(t, r):=\left(\frac{1+t}{1+r^{2-m}}\right)\left\{r \frac{\partial}{\partial r}\left[\frac{1}{r^{m}}\left(\frac{1}{f}-1\right)\right]\right\}, r>0, \\
& y_{m}(t, 0):=\lim _{r \rightarrow 0} y_{m}(t, r) .
\end{aligned}
$$

We can extract $\lambda_{1}$ from the relation

$$
\frac{y_{m}}{1+t}=\frac{r^{2} f}{\left(r^{m}+r^{2}\right)}\left(\frac{m}{(1+f)} \lambda_{2}-\lambda_{1}\right) .
$$


Notice that $y_{m}(t, r) \rightarrow 0$ as $r \rightarrow 0$ whenever $m<2$. Calculating from (4.18), we find that $y_{m}(t, r)$ obeys

$$
\begin{aligned}
\frac{\partial y_{m}}{\partial t}= & \frac{1}{f^{2}} \frac{\partial^{2} y_{m}}{\partial r^{2}}+\frac{1}{r} \alpha_{m} \frac{\partial y_{m}}{\partial r} \\
& +\frac{1}{r^{2}}\left\{\left[\frac{2}{f}\left(2(m-1) r^{m}+m r^{2}\right) y_{m}+1\right] \frac{y_{m}}{1+t}\right. \\
& \left.+\beta_{m} y_{m}+(1+t) \gamma_{m}\right\}
\end{aligned}
$$

where some of the coefficients have rather lengthy expressions so we have introduced the abbreviations

$$
\begin{aligned}
\alpha_{m}:= & \frac{2\left(r^{m}+r^{2}\right)}{f} \frac{y_{m}}{(1+t)}+\frac{4 m-3}{f^{2}}-\frac{2 m}{f}+n-2 \\
& -\frac{2(m-2) r^{2-m}}{f^{2}\left(1+r^{2-m}\right)}, \\
\beta_{m}:= & \frac{7 m^{2}-14 m+4}{f^{2}}-\frac{m(6 m-8)}{f}+(n-2)\left(m-1-\frac{3}{f^{2}}\right) \\
& +\frac{(m-2) r^{2-m}}{1+r^{2-m}}\left[-\frac{(3 m-2)}{f^{2}}+\frac{2 m}{f}-(n-2)\right], \\
\gamma_{m}:= & \frac{1}{\left(r^{m}+r^{2}\right)}\left(\frac{1}{f}-1\right)\left\{\frac{2 m(m-1)(m-2)}{f^{2}}+\frac{2 m^{2}(2-m)}{f}\right. \\
& \left.+(n-2)\left[-m+\frac{m+2}{f}+\frac{2(1-m)}{f^{2}}\right]\right\} .
\end{aligned}
$$

We now claim that the $y_{m}(t, r)$ are bounded below on $\left[0, T_{M}\right) \times[0, \infty)$ by a constant that is independent of $m$. Proceeding in our now usual fashion, let $T$ be such that $0<T<T_{M}$ and define $D:=[0, T] \times[0, \infty)$. As usual, because $y_{m}$ tends to zero for $r \rightarrow \infty$ and for $r \rightarrow 0$, either zero is the lower bound or

$$
\inf _{D} y_{m}=: Y=y_{m}\left(t_{0}, r_{0}\right)<0
$$

for some $t_{0}$ and some $r_{0}>0$. In the latter case, either $t_{0}=0$ and therefore the minimum depends only on initial data $a(r)=f(0, r)$ and not on $m$ or 
Rotationally symmetric Ricci flow on asymptotically flat manifolds 561

$T$, or it occurs at some $t_{0} \in(0, T]$ and then the minimum obeys a quadratic inequality which we now state:

Lemma 5.12. Let $y_{m}$ be defined on $[0, T] \times[0, \infty), T<T_{M}$, by Definition 5.11. For $m<2$, if $y_{m}$ has a negative infimum $Y<0$, then this infimum is realized as a minimum at some $\left(t_{0}, r_{0}\right)$ where $r_{0}>0$ and either $t_{0}=0$ or

$$
\frac{2(m-1)}{f\left(t_{0}, r_{0}\right)} Y^{2}+\left(\frac{1+t_{0}}{r_{0}^{m}+r_{0}^{2}}\right)\left[\left(1+\beta_{m}\left(t_{0}, r_{0}\right)\right) Y+\left(1+t_{0}\right) \gamma\left(t_{0}, r_{0}\right)\right]<0 .
$$

Proof. As discussed immediately above, a negative infimum must be realized at some $\left(t_{0}, r_{0}\right)$ where $r_{0}>0$. Then it follows by applying standard minimum principle arguments to equation (5.27) that either the minimum occurs at $t_{0}=0$ or the nonderivative terms in (5.27) are governed by the inequality

$$
\begin{aligned}
0> & \frac{2}{f\left(t_{0}, r_{0}\right)}\left(2(m-1) r_{0}^{m}+m r_{0}^{2}\right) Y^{2}+Y \\
& +\left(1+t_{0}\right)\left[\beta_{m}\left(t_{0}, r_{0}\right) Y+\left(1+t_{0}\right) \gamma_{m}\left(t_{0}, r_{0}\right)\right] .
\end{aligned}
$$

But in the first term on the right-hand side, use that $\left(2(m-1) r_{0}^{m}+m r_{0}^{2}\right)$ $Y^{2}>2(m-1)\left(r_{0}^{m}+r_{0}^{2}\right) Y^{2}$ for $m<2$ to replace the former by the latter. Replace the second term (the singleton $Y$ ) by $\left(1+t_{0}\right) Y<Y$. These replacements preserve the inequality. Divide by $r_{0}^{m}+r_{0}^{2}$ to complete the proof.

Now further restrict $m$ to some range of form $1<\kappa \leq m<2$, so that the coefficient of $Y^{2}$ in (5.32) is not arbitrarily small; for definiteness $\kappa=$ $3 / 2 \leq m<2$ will do nicely. Then since the criterion (5.32) is quadratic in $Y$ with positive coefficient of $Y^{2}$, it will be violated for $Y$ sufficiently negative. Thus $Y$ cannot be arbitrarily negative, giving a bound on $y_{m}$ expressed in terms of the coefficients in (5.32). It remains therefore to manipulate these coefficients to produce a bound that is manifestly independent of $m$ and $T$. The proof is an exercise in elementary manipulation, but we will give the main points.

Proposition 5.13. Let $3 / 2 \leq m<2$. Then for each $m$, the $y_{m}$ are bounded below on $\left[0, T_{M}\right) \times[0, \infty)$ by an $m$-independent constant.

Proof. As usual, we work on $t \in[0, T]$ with $T<T_{M}$ to obtain a bound which does not depend on $m$ or $T$ and then take $T \rightarrow T_{M}$ when we are done. 
If the lower bound is zero, which occurs at $r_{0}=0$ and as $r_{0} \rightarrow \infty$, then obviously it is independent of $m$ and $T$, so assume that the lower bound is negative. Then it is realized as a minimum at some $\left(t_{0}, r_{0}\right) \in[0, T] \times[0, \infty)$. If $t_{0}=0$, the lower bound is given by the initial data, so again it is clearly $m$ - and $T$-independent. Therefore, assume $t_{0}>0$. Then the criterion (5.32) applies.

In this last case, we start with (5.32) and seek to re-express, where possible, factors of the form $\left(1+t_{0}\right) /\left(r_{0}^{m}+r_{0}^{2}\right)$ in terms of the bounded quantity $u_{m}=\left((1+t) /\left(r^{m}+r^{2}\right)\right)\left(\left(1 / f^{2}\right)-1\right)$. The boundedness of this quantity is described in Remark 5.6; since $f$ is also bounded, we can also make use of equivalent form $(f / 1+f) u_{m}=\left((1+t) /\left(r^{m}+r^{2}\right)\right)((1 / f)-1)$. For example, the term in (5.32) that is constant in $Y$ can be written as (understanding all quantities to be evaluated at $\left.\left(t_{0}, r_{0}\right)\right)$

$$
\begin{aligned}
\frac{\left(1+t_{0}\right)^{2}}{r_{0}^{m}+r_{0}^{2}} \gamma_{m}= & \left(\frac{f u_{m}}{1+f}\right)^{2}\left[2 m(m-2)\left(\frac{m-1}{f}-1\right)\right. \\
& \left.-(n-2)\left(\frac{2(m-1)}{f}+m-4\right)\right] \\
& -2(m-2)(m+n-2)\left(\frac{1+t_{0}}{r_{0}^{m}+r_{0}^{2}}\right) \frac{f u_{m}}{1+f} .
\end{aligned}
$$

We can minimize the term proportional to $u_{m}^{2}$ over $(3 / 2) \leq m \leq 2$. In the second term, note that the coefficient $-2(m-2)(m+n-2)$ is positive for $3 / 2 \leq m<2$. Therefore we write $-2(m-2)(m+n-2)\left(f u_{m} /(1+f)\right) \geq$ $((1 / 2)-n)(f /(1+f))\left|u_{m}\left(t_{0}, r_{0}\right)\right| \geq((1 / 2)-n)(f /(1+f)) C_{u} \geq$ $((1 / 2)-n) C_{u}$, using (5.17). This yields

$$
\begin{aligned}
\frac{\left(1+t_{0}\right)^{2}}{r_{0}^{m}+r_{0}^{2}} \gamma_{m} \geq & \left(\frac{f u_{m}}{1+f}\right)^{2}\left[-\frac{4}{3 \sqrt{3} f}-2(n-2)\left(1-\frac{1}{f}\right)\right] \\
& +\left(\frac{1}{2}-n\right) C_{u}\left(\frac{1+t_{0}}{r_{0}^{m}+r_{0}^{2}}\right) \\
\geq & -k_{1}+\left(\frac{1}{2}-n\right) C_{u}\left(\frac{1+t_{0}}{r_{0}^{m}+r_{0}^{2}}\right),
\end{aligned}
$$

where $k_{1}$ is a (positive) constant independent of $m, T$, and $Y{ }^{6}$ The second term still contains an unwanted factor of $\left(1+t_{0}\right) /\left(r_{0}^{m}+r_{0}^{2}\right)$ with negative coefficient, but for $Y$ sufficiently negative we will be able to dominate this

\footnotetext{
${ }^{6}$ For example, $k_{1}=C_{u}^{2}\left[\left(1 / C_{f}^{-}\right)+2(n-2)\right]$ would do fine, where we write $C_{f}^{-}:=$ $\sqrt{C_{f^{2}}^{-}}$.
} 
Rotationally symmetric Ricci flow on asymptotically flat manifolds 563

term with positive contributions coming from the part of the criterion (5.32) that is linear in $Y$.

To examine the linear term, start from the expression

$$
\begin{aligned}
\left(\frac{1+t_{0}}{r_{0}^{m}+r_{0}^{2}}\right)\left(1+\beta_{m}\right) Y= & \left(\frac{1+t_{0}}{r_{0}^{m}+r_{0}^{2}}\right)\left\{1+\frac{7 m^{2}-14 m+4}{f^{2}}-\frac{m(6 m-8)}{f}\right. \\
& +(n-2)\left(m-1-\frac{3}{f^{2}}\right) \\
& \left.+\frac{(2-m) r^{2-m}}{1+r^{2-m}}\left[\frac{3 m-2}{f^{2}}-\frac{2 m}{f}+n-2\right]\right\} Y .
\end{aligned}
$$

The terms in the last line simplify since we can use that $Y<0,(3 / 2) \geq m<2$, and $n \geq 3$ to write

$$
\begin{aligned}
\frac{(2-m) r^{2-m}}{1+r^{2-m}}\left[\frac{3 m-2}{f^{2}}-\frac{2 m}{f}+n-2\right] Y & >\frac{(2-m) r^{2-m}}{1+r^{2-m}}\left[\frac{3 m-2}{f^{2}}+n-2\right] Y \\
& >(2-37) \quad\left[\frac{3 m-2}{f^{2}}+n-2\right] Y .
\end{aligned}
$$

Now we can combine this result with (5.36) and again absorb the factor of $\left(1+t_{0}\right) /\left(r_{0}^{m}+r_{0}^{2}\right)$, wherever possible, using $u_{m}$. We get

$$
\begin{aligned}
\left(\frac{1+t_{0}}{r_{0}^{m}+r_{0}^{2}}\right)\left(1+\beta_{m}\right) Y> & \frac{f u_{m}}{(1+f)}\left[\frac{4 m^{2}-6 m-3(n-2)}{f}\right. \\
& \left.-2 m^{2}+2 m-3(n-2)\right] Y \\
& -\left(2 m^{2}-2 m+2 n-5\right)\left(\frac{1+t_{0}}{r_{0}^{m}+r_{0}^{2}}\right) Y \\
\geq & \frac{f u_{m}}{(1+f)}\left[\frac{4 m^{2}-6 m-3(n-2)}{f}\right. \\
& \left.-2 m^{2}+2 m-3(n-2)\right] Y \\
& -\left(2 n-\frac{7}{2}\right)\left(\frac{1+t_{0}}{r_{0}^{m}+r_{0}^{2}}\right) Y
\end{aligned}
$$

where in the last line minimized over $3 / 2 \leq m<2$. It is again evident that this is the sum of a bounded term and a term involving $\left(1+t_{0}\right) /\left(r_{0}^{m}+r_{0}^{2}\right)$. 
Both these terms are linear in $Y$. That is,

$$
\left(\frac{1+t_{0}}{r_{0}^{m}+r_{0}^{2}}\right)\left(1+\beta_{m}\right) Y \geq k_{2} Y-\left(2 n-\frac{7}{2}\right)\left(\frac{1+t_{0}}{r_{0}^{m}+r_{0}^{2}}\right) Y,
$$

where $k_{2}$ is a constant independent of $m, T$, and $Y .7$

Inserting (5.35) and (5.39) into the criterion (5.32) and using that $\left(2(m-1) / f\left(t_{0}, r_{0}\right)\right) Y^{2} \geq\left(3 / f\left(t_{0}, r_{0}\right)\right) Y^{2}$ for $3 / 2 \leq m<2$, we obtain the following necessary condition for $Y<0$ to be the minimum of $y_{m}\left(t_{0}, r_{0}\right)$ at some $t_{0}>0$ :

$$
\begin{aligned}
0 \geq & \frac{3}{f\left(t_{0}, r_{0}\right)} Y^{2}+k_{2} Y-k_{1} \\
& +\left[\left(\frac{1}{2}-n\right) C_{u}-\left(2 n-\frac{7}{2}\right) Y\right]\left(\frac{1+t_{0}}{r_{0}^{m}+r_{0}^{2}}\right) .
\end{aligned}
$$

Then a necessary condition for $Y<(1-2 n / 4 n-7) C_{u}$ to be the minimum of $y_{m}\left(t_{0}, r_{0}\right)$ at some $t_{0}>0$ is

$$
0>\frac{3}{f\left(t_{0}, r_{0}\right)} Y^{2}+k_{2} Y-k_{1}
$$

which is clearly violated whenever

$$
Y<C_{Y}:=\min \left\{\left(\frac{1-2 n}{4 n-7}\right) C_{u},-\frac{C_{f}^{+}}{6}\left[k_{2}+\sqrt{k_{2}^{2}+\frac{12}{C_{f}^{-}} k_{1}}\right]\right\}
$$

where we use the short-hand $C_{f}^{ \pm}:=\sqrt{C_{f^{2}}^{ \pm}}$. We conclude that

$$
y_{m} \geq C_{y}^{-}:=\min \left\{C_{Y}, \inf _{r}\left\{y_{m}(0, r)\right\}\right\}
$$

on $[0, T] \times[0, \infty)$ and since these bounds do not depend on $T$, taking $T \rightarrow$ $T_{M}$ we see that they hold as well on $\left[0, T_{M}\right) \times[0, \infty)$.

Corollary 5.14. There is a constant $C_{\lambda_{1}}^{+}$such that

$$
\lambda_{1}(t, r) \leq \frac{C_{\lambda_{1}}^{+}}{1+t}
$$

on $\left[0, T_{M}\right) \times[0, \infty)$.

\footnotetext{
${ }^{7}$ For example, from elementary considerations applied to (5.38) we obtain that $k_{2}=8 C_{u}$ is a suitable bound.
} 
Rotationally symmetric Ricci flow on asymptotically flat manifolds 565

Proof. First we prove that $y_{2}$ is bounded below by $C_{y}^{-}$. As with Corollaries 5.3 and 5.7, the map $m \mapsto y_{m}(t, r)$, with fixed $t$ and fixed $r>0$, is continuous, so the bound (5.43) applies to $y_{2}(t, r)$ except possibly at $r=0$. Then the continuity of $y_{2}$ at $r=0$ implies that the bound holds there as well.

Next, the $m=2$ case of (5.26) yields

$$
\lambda_{1}=\frac{2}{1+f} \lambda_{2}-\frac{y_{2}}{1+t} .
$$

Using (5.18) and the facts that $C_{y}^{-} \leq 0$ and $C_{v}^{+} \geq 0$, we can write this as

$$
\begin{aligned}
\lambda_{1}(t, r) & \leq\left(\frac{2}{1+f}\right) \frac{2 C_{v}^{+}}{(1+t) C^{-}+f^{2}}-\frac{2 C_{y}^{-}}{(1+t) f} \\
& \leq \frac{1}{1+t}\left[\frac{4 C_{v}^{+}}{C_{f^{2}}^{-}}-\frac{2 C_{y}^{-}}{C_{f}^{-}}\right],
\end{aligned}
$$

where we have used that $0<C_{f^{2}}^{-} \leq f^{2}$ and $C_{f}^{-}:=\sqrt{C_{f^{2}}^{-}}$. Now let $C_{\lambda_{1}}^{+}$equal the quantity in square brackets in the last line.

We can now prove the main theorem.

\subsection{Proof of Theorem 1.1}

Proof of Statement (i). By Corollaries 5.3, 5.7, 5.10, and 5.14, the sectional curvatures in $\left[0, T_{M}\right) \times[0, \infty)$ are bounded above and below by bounds of the form

$$
\left|\lambda_{1,2}\right| \leq \frac{\left|C_{\lambda_{1,2}}^{ \pm}\right|}{1+t} \leq\left|C_{\lambda_{1,2}}^{ \pm}\right|
$$

Thus, by Theorem 4.7, we can take $T_{M}=\infty$ and can conclude that there is a constant $C_{0}$ such that

$$
\sup _{x \in \mathbb{R}^{n}}|\overline{\operatorname{Rm}}(x, t)|_{\bar{g}(t, x)} \leq \frac{C_{0}}{1+t} \forall t \geq 0 .
$$

This proves the existence for all $t \in[0, \infty)$ of the solution developing from the initial condition, Statement (i) of the theorem and also the $\ell=0$ estimate of (iii).

Proof of Statement (ii). This is immediate from Theorem 3.1.

Proof of Statement (iii). Follows directly from (5.48) and [11, Theorem 7.1]. 
Proof of Statement (iv). This follows from the Compactness Theorem 1.2 of [12] and statement (iii), provided the injectivity radius at the origin is $>\delta \geq 0$ for some $\delta$ independent of $t$. Since the metric is uniformly equivalent to the Euclidean metric and the sectional curvatures are uniformly bounded in time, this follows immediately from, for example, the Cheeger-GromovTaylor injectivity radius estimate [3, Theorem 4.7]. ${ }^{8}$

Proof of Statement (v). Immediate from Remark 3.3.

\section{Acknowledgements}

We thank Suneeta Vardarajan for discussions concerning the string theory motivation for this work. E.W. also thanks Barton Zwiebach for his explanation of the rolling tachyon. This work was begun during a visit by T.A.O. to the Dept of Mathematical and Statistical Sciences of the University of Alberta, which he thanks for hospitality. The work was partially supported by a discovery grant from the Natural Sciences and Engineering Research Council of Canada.

\section{References}

[1] S. Angenent and D. Knopf, An example of neckpinching for Ricci flow on $S^{n+1}$, Math. Res. Lett. 11 (2004), 493-518.

[2] R. Bartnik, The mass of an asymptotically flat manifold, Comm. Pure Appl. Math. 39 (1986), 661-693.

[3] J. Cheeger, M. Gromov and M. Taylor, Finite propagation speed, Kernel estimates for functions of the Laplace operator, and the geometry of complete Riemannian manifolds, J. Diff. Geom. 17 (1982), 15.

[4] B. L. Chen and X. P. Zhu, Uniqueness of the Ricci flow on complete noncompact manifolds, Preprint 2005, arxiv.org:math.DF/0505447.

[5] M. Choptuik, Talk given at 15th International Conference on General Relativity and Gravitation (GR15), Pune, India, 16-21 December 1997, Preprint 1998, arxiv:gr-qc/9803075.

\footnotetext{
${ }^{8}$ Even more simply, since the constant- $r$ surfaces are convex throughout the flow, there are no closed geodesics. Then it follows from the sectional curvature bound (5.48) that $\operatorname{inj}\left(\mathbb{R}^{n}, g(t)\right) \geq(\pi \sqrt{1+t}) / \sqrt{C_{0}}$. Since this gives a global bound on the injectivity radius, less powerful convergence theorems (e.g., [22, Theorem 7.1.3]) suffice to finish the proof.
} 
Rotationally symmetric Ricci flow on asymptotically flat manifolds 567

[6] X. Dai and L. Ma, Mass under the Ricci flow, Preprint 2005, arxiv.org:math.DG/0510083.

[7] D. H. Friedan, Nonlinear Models in $2+\varepsilon$ Dimensions, PhD thesis, University of California, Berkeley, 1980 (unpublished); Phys Rev Lett 45 (1980), 1057-1060; Ann. Phys. (NY) 163 (1985), 318-419.

[8] D. Garfinkle and J. Isenberg, Critical behavior in Ricci flow, Preprint 2003, arxiv:math.DG/0306129.

[9] M. Gutperle, M. Headrick, S. Minwalla and V. Schomerus, Space-time energy decreases under world sheet $R G$ flow, JHEP 0301 (2003) 073, arxiv:hep-th/0211063.

[10] R. S. Hamilton, Three-manifolds with positive Ricci curvature, J. Diff. Geom. 17 (1982), 255-306.

[11] R. S. Hamilton, The formation of singularities in the Ricci flow, Surveys in differential geometry, vol. 2, International Press, Cambridge, MA, $1995,7-136$.

[12] R. S. Hamilton, A compactness property for solutions of the Ricci flow, Amer. J. Math. 117 (1995), 545-572.

[13] M. Headrick, S. Minwalla and T. Takayanagi, Closed string tachyon condensation: an overview, Class Quantum Gravit 21 (2004), S1539S1565, arxiv:hep-th/0405064.

[14] T. A. Ivey, The Ricci flow on radially symmetric $\mathbb{R}^{3}$, Comm. Partial Diff. Eqns 19 (1994), 1481-1500.

[15] B. List, Evolution of an extended Ricci flow system, $\mathrm{PhD}$ thesis, Max-Planck-Institut für Gravitationsphysik and der Freien Universität Berlin, unpublished.

[16] T. A. Oliynyk, An existence proof for the gravitating BPS monopole, Ann. Henri Poincaré 7 (2006), 199-232.

[17] T. Oliynyk, V. Suneeta and E. Woolgar, Irreversibility of world-sheet renormalization group flow, Phys. Lett. B610 (2005), 115-121, arxiv: hepth/0410001.

[18] T. Oliynyk, V. Suneeta and E. Woolgar, A gradient flow for worldsheet nonlinear sigma models, Nuc. Phys. B739 (2006), 441-458, arxiv:hepth/0510239. 
[19] T. A. Oliynyk and E. Woolgar, Asymptotically flat Ricci flows, Preprint, math.DG/0607438.

[20] G. Perelman, The entropy formula for the Ricci flow and its geometric applications, preprint 2002, arxiv:math.DG/0211159; Ricci flow with surgery on three-manifolds, Preprint 2003, arxiv:math.DG/0303109.

[21] W. Shi, Deforming the metric on complete Riemannian manifolds, J. Diff. Geom. 30 (1989), 223-301.

[22] P. Topping, Lectures on the Ricci Flow, LMS Lecture Note Series, Cambridge, 2006, 325.

School of Mathematical Sciences

MONASH UNIVERSITY

Australia VIC 3800

E-mail address: todd.oliynyk@sci.monash.edu.au

Department of Mathematical and Statistical Sciences

University of Alberta

EDMONTON

Alberta

Canada T6G 2G1

E-mail address: ewoolgar@math.ualberta.ca

Received August 1, 2006 